\title{
Integrated Navigation Design Using a Gimbaled Vision/LiDAR System with an Approximate Ground Description Model
}

\author{
Sukchang Yun*, Young Jae Lee**, Chang Joo Kim*** and Sangkyung Sung**** \\ Konkuk University, Seoul, Korea
}

\begin{abstract}
This paper presents a vision/LiDAR integrated navigation system that provides accurate relative navigation performance on a general ground surface, in GNSS-denied environments. The considered ground surface during flight is approximated as a piecewise continuous model, with flat and slope surface profiles. In its implementation, the presented system consists of a strapdown IMU, and an aided sensor block, consisting of a vision sensor and a LiDAR on a stabilized gimbal platform. Thus, two-dimensional optical flow vectors from the vision sensor, and range information from LiDAR to ground are used to overcome the performance limit of the tactical grade inertial navigation solution without GNSS signal. In filter realization, the INS error model is employed, with measurement vectors containing two-dimensional velocity errors, and one differenced altitude in the navigation frame. In computing the altitude difference, the ground slope angle is estimated in a novel way, through two bisectional LiDAR signals, with a practical assumption representing a general ground profile. Finally, the overall integrated system is implemented, based on the extended Kalman filter framework, and the performance is demonstrated through a simulation study, with an aircraft flight trajectory scenario.
\end{abstract}

Key words: Vision/LiDAR, navigation, ground slope model, integrated system, extended Kalman filter

\section{Introduction}

One of the most essential technologies for operating unmanned vehicles is to compute navigation information, including the position, velocity, and attitude of a vehicle. A number of autonomous vehicles have been developed, such as a guided weapon system, UGV (Unmanned Ground Vehicle), USV (Unmanned Surface Vehicle), and UAV (Unmanned Aerial Vehicle), for surveillance, reconnaissance, and many other dangerous and difficult missions. For autonomously operating these unmanned vehicles, many navigation sensors and systems are used, for example IMU (Inertial Measurement Unit), GNSS (Global Navigation Satellite System), vision sensor, LiDAR (Light Detection and Ranging), magnetometer, barometer, etc. Among these, GNSS is an essential system, which can provide accurate absolute position information of a vehicle within certain error bounds, as well as velocity and time information. In particular, the
GPS/INS integrated system, which can provide a drift-free and accurate solution of a highly dynamic vehicle, by using GPS (Global Positioning System) satellite signals to correct the solution from an Inertial Navigation System (INS), has been widely used on ground and aerial vehicles, for precise navigation purposes.

However, the performance of the GNSS-based navigation system is likely to be affected by satellite observation environments, since the system cannot provide the navigation solution in poor environments. For example, when a vehicle is downtown or in cloud forests, the number of visible satellites decreases drastically, and multipath error is very likely to occur. Furthermore, under indoor or jamming environments, the satellite navigation system cannot be used. To overcome the unavailability problem of GNSS, various researches have been studied. Among those, research on the integration of IMU and vision sensor is notable [1-3]. The research is generally based on SLAM (Simultaneous Localization and Mapping)
This is an Open Access article distributed under the terms of the Creative Commons Attribution Non-Commercial License (http://creativecommons.org/licenses/by$\mathrm{nc} / 3.0 /$ which permits unrestricted non-commercial use, distribution, and reproduction in any medium, provided the original work is properly cited.

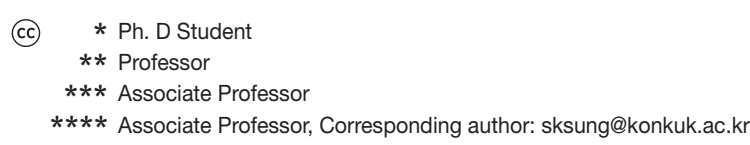


technologies. The most representative way is to use feature points, which are easily distinguishable from other pixel points of the captured image [4-9]. The navigation solution of the feature point-based SLAM system has generally a diverging property, since the navigation algorithm inherently relies on the accuracy of both the feature point position estimates, and vehicle estimates, which tend to deteriorate, due to mutual error accumulations. Also, it is difficult to detect and track high-quality feature points in unstructured environments where no previously known landmark exists.

Meanwhile, an optical flow that represents the motion of pixels was also utilized in vision-aided navigation systems [10, 11, 13-14]. In [11], optical flow vectors were used for road navigation, to estimate the forward velocity and heading angle of a car, and detect moving cars on the road. Additionally, it was applied to the autonomous flight control of a MAV (Micro Air Vehicle) in indoor corridor environments, and autonomous take-off and landing of a small-scale UAV $[13,14]$. However, it is rarely reported that optical flow is directly adopted for estimating 6 DoF (Degree of Freedom) navigation solutions.

In a different approach from vision-based navigation systems, the LiDAR-based navigation system has been independently developed to deal with GNSS-denied environments. As a result, a localization and mapping system for mobile robots and multi-rotors has been developed. In [15], scan matching based two-dimensional SLAM and INS are loosely integrated, based on an EKF (extended Kalman filter) framework, and the developed system is applied to UAV, USV and small handheld embedded systems. However, the designed system considers two-dimensional motion only in the limited environment where specific obstacles are within the range of LiDAR. Thus, it cannot be applied in unstructured outdoor environments, such as open terrain.

In a few works, research on SLAM combining both vision and LiDAR sensor were investigated. For instance, IR scanner, vision sensors and encoders were simultaneously integrated, based on a particle filter framework for mobile robot localization, with the assumption of previously known map information. Besides, research on detecting moving cars and pedestrians, and research on indoor path planning of UAV were developed, by combining computer vision processing results with LiDAR measurements [16-18]. Nevertheless, these efforts put most emphasis on detecting and localizing possible obstacles via heterogeneous sensor fusion, and thus suffered from generating extensive status information of the host vehicles. Consequently, a literature survey reveals that no published result presents direct integration of vision sensor and LiDAR within an INS mechanization scheme, for a complete navigation solution for unstructured outdoor environments.

In this paper, we present a Vision/LiDAR aided integrated navigation system, for an environment where 1) GNSS signals cannot be used; 2) the altitude of the ground surface is varying; and 3) there is no known map information. The presented system consists of a mid grade strapdown IMU and a vision/LiDAR system, on a gimbaled platform, onboard the vehicle. The system turns out to provide a reliable navigation solution, by suppressing divergence of the INS error, with the help of vision sensor and LiDAR measurements. In detail, the horizontal velocity error is obtained by correcting the velocity estimate of INS, with that derived from optical flow measurements. In particular, a new piecewise continuous slope model is introduced for the ground, in calculating altitude increment of the proposed measurement model. Basically, range error is obtained by combining the INS solution and attitude compensated range information from LiDAR. In parallel, the height of the ground surface is estimated, which relates to the geometric relation between two boundary LiDAR measurements. Then, the combined error, including range error and ground altitude variation, serves as the true measurement of the vehicle's altitude change. Finally, the overall integrated navigation system is implemented, based on EKF framework, supported by a simulation study to verify the feasibility of the proposed system.

The rest of the paper is organized as follows: Section 2 describes the concept of the proposed integrated navigation and its coordinate system. In Section 3, the INS error model and sensor model are presented, for constructing the integrated navigation filter. After that, Section 4 provides simulation results, to verify its feasibility in GNSS-denied environments. Finally, Section 5 concludes the paper, with a discussion of our current research.

\section{System Illustration}

The proposed integrated navigation system considers its operation onboard a UAV, flying over flat or sloped ground, under GNSS-denied environments. To illustrate the working principle and basic architecture of the proposed system, the sensor integration concept and the adapted coordinate system for the low or mid grade IMU (inertial measurement unit), vision sensor, and LiDAR are presented in this section.

\subsection{Concept of the Proposed System}

The concept of the proposed integrated navigation system is described in Fig. 1. The system provides a relative 
navigation solution, with the aid of the vision sensor and LiDAR measurements, when the GNSS signal cannot be used. The IMU operating at a high rate is mounted on the vehicle body, which results in a strapdown INS mechanization. The vision sensor and LiDAR operating at a lower rate than the IMU are mounted on a stabilized twodimensional gimbal system; thus these sensors can keep their sensing axis perpendicular to the local navigation frame, regardless of the vehicle's roll/pitch motion. Although the GNSS/INS integrated system guarantees a tolerable navigation accuracy in normal conditions, the performance abruptly degrades, as GNSS signal outage occurs. In this background, it is attempted to suppress the rapid divergence of the navigation solution, by compensating INS errors, through the measurements from the vision sensor and LiDAR.

Optical flow vectors representing the motion of pixels are calculated, using consecutive image frames. If it is assumed that the rotational motion of the vehicle is sufficiently negligible, compared with the longitudinal or lateral motion, the optical flow effectively represents the translational motion of the vehicle, because the motion of the vision sensor is not affected by the roll/pitch motion. Therefore, by associating the flow vector with the camera model for coordinate transformation, the horizontal translational velocity can be computed. At the same time, the altitude variation of the ground surface can be calculated, using two range measurements (forward and backward range signal) from the LiDAR, which can be further used to obtain the vehicle's accurate altitude change on a general sloped ground.

\subsection{Sensor Coordinate Systems}

The sensor coordinate system of the three sensors for constructing the presented navigation system is shown in Fig. 2.

The coordinate system of the IMU is equal to the body frame of the vehicles. The vision sensor and LiDAR are mounted on the two-dimensional gimbal system, which is referenced to the gimbal coordinate system, and aligned with the local navigation frame (NED frame), except for the down axis. In the figure below, the subscript $\mathbf{b}$ denotes the body-fixed coordinate system, and the subscript $\mathrm{g}$ denotes the gimbal coordinate system. Images from the vision sensor are on the $X_{g}-Y_{g}$ plane, and the LiDAR provides detection ranges and scan angles on the $X_{g}-Z_{g}$ scan plane, whose zero degrees scan angle is equal to the $\mathrm{Z}_{\mathrm{g}}$ axis. Then, the relationship between body-fixed coordinate and gimbal coordinate system can be simply expressed, using the wellknown direction cosine matrix, containing only roll $\left(a_{\phi}\right)$ and pitch angle $\left(a_{\theta}\right)$ :

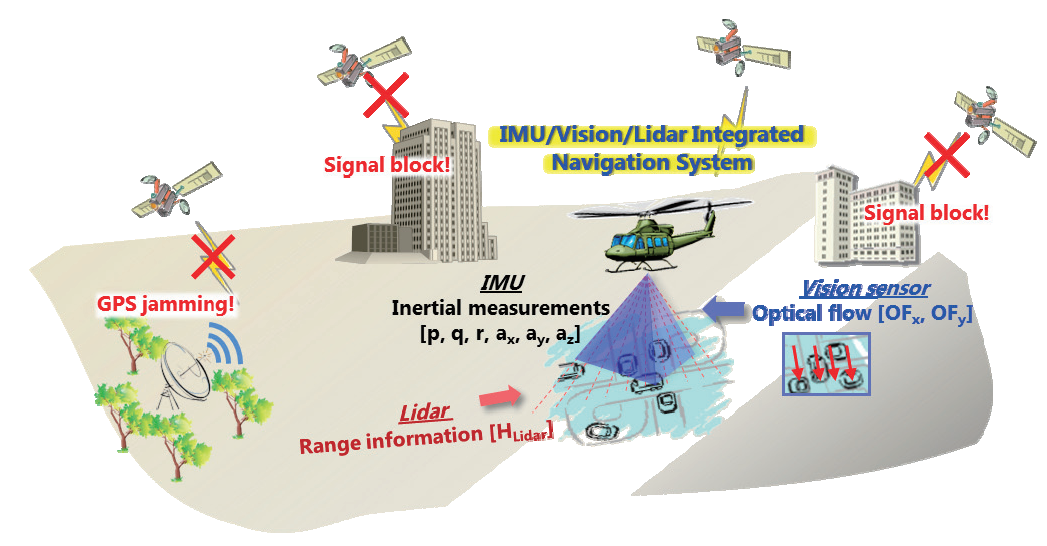

Fig. 1. System concept of the proposed system

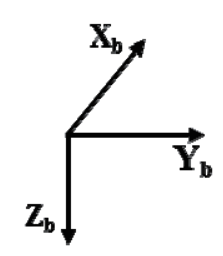

(a) IMU

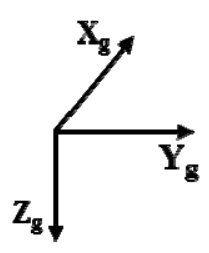

(b) vision sensor

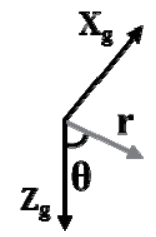

(c) LiDAR

\section{r: detection range}

$\boldsymbol{\theta}$ : scan angle

Fig. 2. Coordinate systems among sensors 


$$
C_{b}^{g}=\left[\begin{array}{ccc}
\cos \left(a_{\theta}\right) & \sin \left(a_{\phi}\right) \sin \left(a_{\theta}\right) & \cos \left(a_{\phi}\right) \sin \left(a_{\theta}\right) \\
0 & \cos \left(a_{\phi}\right) & -\sin \left(a_{\phi}\right) \\
-\sin \left(a_{\theta}\right) & \sin \left(a_{\phi}\right) \cos \left(a_{\theta}\right) & \cos \left(a_{\phi}\right) \cos \left(a_{\theta}\right)
\end{array}\right],
$$

\section{Integrated Navigation System}

\subsection{INS Error Model}

The Psi angle approach is used as the INS error model. The error model is the most widely adopted approach, and has been implemented in many tightly coupled integrated systems [19]. The INS error model is shown below.

$$
\begin{aligned}
& \delta \dot{\mathbf{v}}=-\left(\omega_{i e}+\omega_{i n}\right) \times \delta \mathbf{v}-\delta \boldsymbol{\psi} \times \mathbf{f}+\nabla \\
& \delta \dot{\mathbf{r}}=-\omega_{e n} \times \delta \mathbf{r}+\delta \mathbf{v} \\
& \delta \dot{\psi}=-\omega_{i n} \times \delta \boldsymbol{\psi}+\varepsilon
\end{aligned}
$$

where,

$\delta \mathbf{r}, \delta \mathbf{v}, \delta \psi$ : Position, velocity, attitude error vectors w.r.t. the navigation frame

$\omega_{i e}$ : Earth rate vector

$\omega_{i n}$ : Angular rate vector of the true coordinate system w.r.t. the inertial frame

$\omega_{e n}$ : Angular rate vector of the true coordinate system w.r.t. the Earth frame

$\nabla:$ Accelerometer bias error vector

$\varepsilon$ : Gyro bias error vector

f : Specific force vector

\subsection{Sensor Measurement Model}

The vision sensor and LiDAR maintain the horizontality condition all the time. Optical flow vectors are modeled using the translation velocity of the vehicle and range information, and the altitude variation is modeled using LiDAR forward/backward range measurements, and their geometrical relations with the ground surface.

\subsubsection{Optical Flow Measurement Model}

The direction of the optical flow vector is opposite to the direction of the vehicle motion, and its magnitude is proportional to the velocity, and inversely proportional to the relative height between the sensor and ground surface. The simple planar model for the translational velocity matches well with most fixed-wing aircraft flight cases, where no high maneuvering dynamics are assumed [12]. Then, equations (3) and (4) represent an optical flow model, based on a pinhole camera model. In this equation, optical flow is the amount of pixel movement in pixel units.

$$
\begin{aligned}
& O F_{x}=\frac{F L}{H \times \text { PixSize }_{x}} \times\left(-V_{x} \times \Delta t\right), \\
& O F_{y}=\frac{F L}{H \times \text { PixSize }_{y}} \times\left(-V_{y} \times \Delta t\right),
\end{aligned}
$$

where,

$O F_{x}, O F_{y}$ : Optical flow measurements [pixel]

$V_{x}, V_{y}$ : Translational velocity w.r.t. the body frame $[\mathrm{m} / \mathrm{s}]$

$\triangle t$ : Interval time [s]

$H$ : Ground height $[\mathrm{m}]$

$F L$ : Camera focal length [m]

PixSize $_{x}$, PixSize $_{y}$ : Magnitude of the 1 pixel [m]

Then, the translational velocity in the NED frame is represented by the following equation:

$$
\left[\begin{array}{c}
V_{x} \\
V_{y}
\end{array}\right]=\left[\begin{array}{cc}
\cos \left(\psi_{D}\right) & \sin \left(\psi_{D}\right) \\
-\sin \left(\psi_{D}\right) & \cos \left(\psi_{D}\right)
\end{array}\right]\left[\begin{array}{c}
V_{N} \\
V_{E}
\end{array}\right],
$$

where, $\psi_{D}$ is the heading angle [rad] of the aircraft. In the case that a moderate maneuvering dynamics of the aircraft is considered, the noise model of the optical flow can be assumed as an additive white Gaussian form.

\subsubsection{Altitude Measurement Model}

LiDAR can measure the distance to targets, with respect to scanning angles. The detected range at a scan angle of

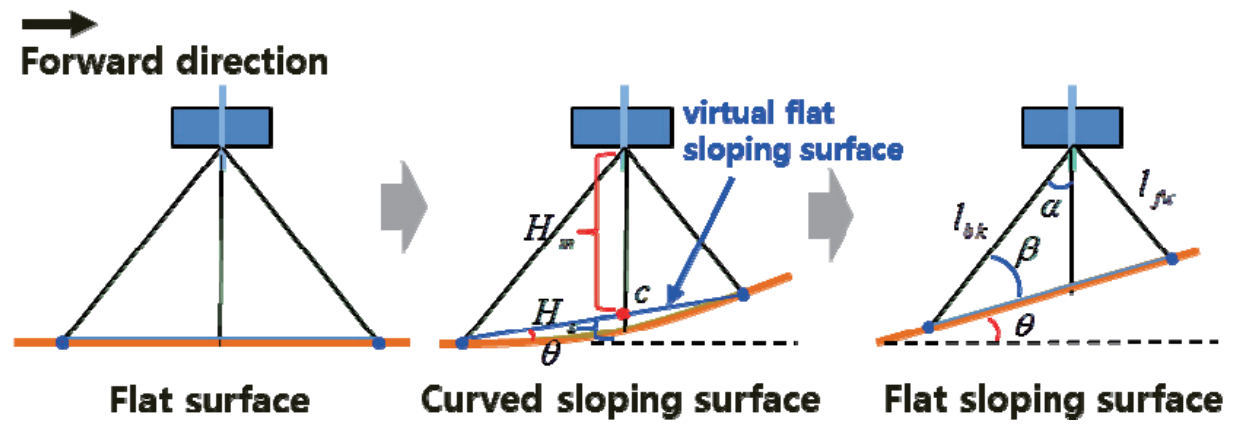

Fig. 3. LiDAR measurements, according to the ground surface type 
zero degrees represents the vertical distance to the ground surface, since the LiDAR always looks downward, as well as the vision sensor. If the height of the ground surface is not varying, the vertical distance can be simply used as an altitude measurement. But if it is varying, the height of the ground surface should be computed, through a geometric condition. Fig. 3 describes the geometrical relations between the LiDAR measurements and the ground surface.

When it is assumed that the shape of the ground surface is smooth, the type of ground surface is categorized into (1) flat surface, (2) curved sloping surface, and (3) flat sloping surface. In the above figure, by using forward range $l_{f w}$ with a scanning angle of $\alpha$ degrees, and backward range $l_{b k}$ with a scanning angle of $-\alpha$ degrees, the angle $\beta$ between the backward range and the sloping surface can be obtained as shown in (5).

$$
\beta=\tan ^{-1}\left(\frac{\sin (\pi-2 \alpha)}{\cos (\pi-2 \alpha)+l_{b k} / l_{f w}}\right),
$$

The slope angle $\theta$ can be calculated as shown in (6). In the case of surface type (2), the slope angle means the angle of the virtual flat sloping surface line connecting two cross points, where the forward/backward measurements and the curved ground surface intersect one another.

$$
\theta=\frac{\pi}{2}-(\alpha+\beta)
$$

In the above figure, $H_{s}$ represents the distance between LiDAR and the virtual surface, and $H_{m}$ represents the height of the virtual surface. Height variation of the virtual ground surface $\left(\triangle H_{s}\right)$ can be calculated, using the forward velocity of the vehicle, and the slope angle. Also, distance variation $\left(\triangle H_{m}\right)$ between the vehicle and the virtual ground surface can be obtained, by differencing the current and previous distance.

$$
\begin{aligned}
\Delta H_{s} & =V_{\text {forward }} \tan (\theta) \Delta t, \\
\Delta H_{m} & =\text { current } H_{m}-\text { previous } H_{m} .
\end{aligned}
$$

Finally, the height variation of the vehicle is computed as

$$
\Delta H=\Delta H_{s}+\Delta H_{m} .
$$

\subsection{Integrated Navigation Filter}

There are various filtering methods, such as the EKF, UKF (unscented Kalman filter), CKF (cubature Kalman filter), and PF (particle filter) for nonlinear system application. In many cases, the EKF approach for the nonlinear estimation problem provides a strong cost-effective result, in comparison with other nonlinear estimation methods. Thus in this paper, considering aspects of algorithm complexity, estimation performance, and onboard hardware implementation, the integrated navigation filter is constructed based on the EKF framework, using the INS error model and sensor measurement models in the previous sections. In this scheme, the linearized system and observation model around the last state estimates are used for each time update, and measurement update computation. Fig. 4 briefly summarizes the formulations of the EKF.

The state vector consists of position, velocity and attitude error of the vehicle; and additionally, bias errors of the IMU measurements are augmented. The state can be expressed as

$$
\mathbf{x}=\left[\begin{array}{lllll}
\delta \mathbf{r} & \delta \mathbf{v} & \delta \boldsymbol{\psi} & \nabla & \varepsilon
\end{array}\right]^{T},
$$

The dynamics of the system model has the following form. Detailed expression for the dynamic matrix, $\mathrm{F}$ is shown in Appendix A.

$$
\dot{\mathbf{x}}=F \mathbf{x}+w,
$$

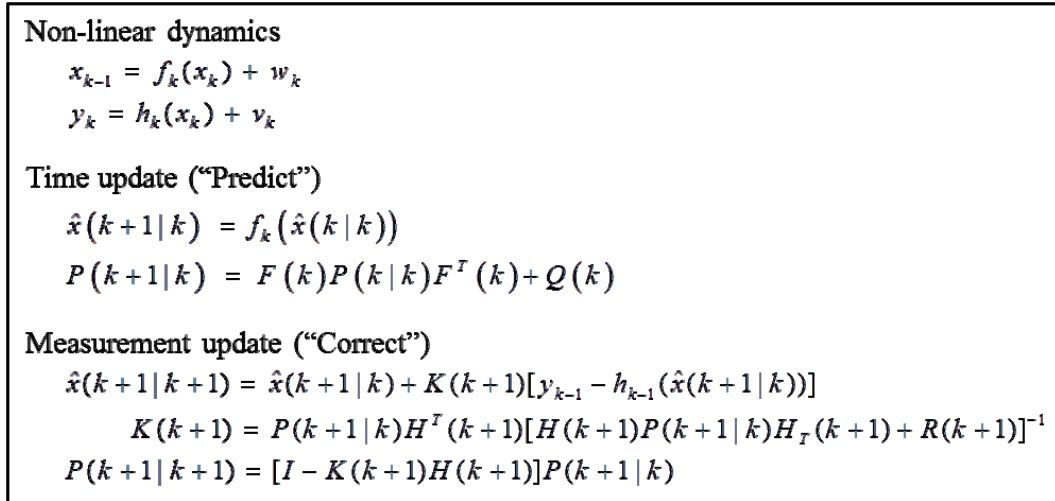

Fig. 4. Summary of the EKF formulations [20] 
where,

$$
F=\left[\begin{array}{ccc}
F_{11} & F_{12} & F_{13} \\
0 & F_{22} & 0 \\
0 & 0 & F_{33}
\end{array}\right]
$$

The observation vector consists of the altitude difference between the altitude estimated from the INS and LiDAR altitude, and horizontal velocity difference with respect to the body frame, between velocity estimated from the INS, and that calculated using optical flow vectors. Thus, the observation vector can be expressed as

$$
\mathbf{z}=\left[\begin{array}{lll}
r_{D}-\hat{r}_{D} & V_{x}-\hat{V}_{x} & V_{y}-\hat{V}_{y}
\end{array}\right]^{T},
$$

where,

$r_{D}$ : Vertical position w.r.t. the navigation frame (NED frame)

$\hat{r}_{D}$ : Computed vertical position w.r.t. the navigation frame, using LiDAR measurements

$\hat{V}_{x}, \hat{V}_{y}$ : Computed horizontal velocity, using optical flow

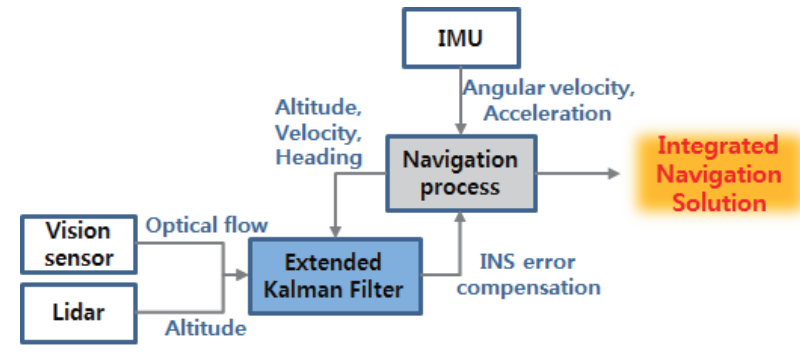

Fig. 5. Integrated navigation filter structure vectors.

The observation model, representing relations between the state vector and the observation vector, is modeled as (12).

$$
\mathbf{z}=H \mathbf{x}+v,
$$

The measurement matrix is represented by

$$
H=\left[\begin{array}{cccc} 
& 1 & 0_{1 \times 3} & \\
0_{3 \times 2} & 0 & C_{n(1-\text { row })}^{b} & 0_{3 \times 9} \\
& 0 & C_{n(2-\text { row })}^{b} &
\end{array}\right]
$$

where, $C_{n}^{b}$ : Transformation matrix from the navigation frame to body frame. In the above models, all process and measurement noises are modeled as zero-mean white gaussian noises.

Fig. 5 represents the structure of the proposed integrated navigation filter. An indirect feedback structure is adopted, to compensate estimated error. In this filter structure, the integrated navigation solution is estimated by compensating the estimated INS error, using the measurement vectors from optical flow and LiDAR.

\section{Simulations}

The feasibility of the proposed integrated navigation filter in the previous section was verified through simulations, by comparing the navigation performance of the proposed

Table 1. Flight profile

\begin{tabular}{cc}
\hline Order & Profile \\
\hline 1 & level flight \\
2 & ascending flight (pitch angle: 10 degree) \\
3 & bank turn (roll angle: 15 degree) \\
4 & descending flight (pitch angle: -10 degree) \\
5 & level flight \\
\hline
\end{tabular}

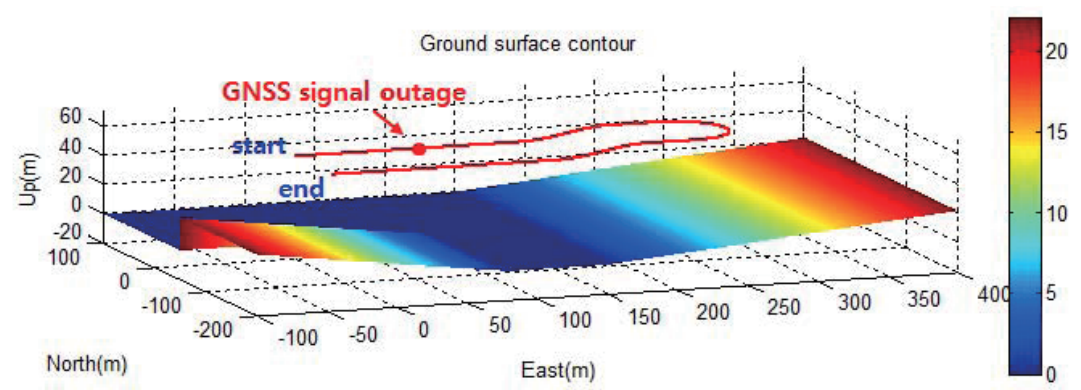

Fig. 6. Height distribution of ground surface, and reference flight trajectory 
system, with that of an INS-only system, when GNSS signal outage occurs.

\subsection{Simulation Environments}

The navigation scenario is that a fixed-wing UAV first makes a level flight with GNSS/INS navigation mode, and then the navigation mode is changed to an integrated navigation system, as soon as GNSS signal outage occurs, at 10 seconds from the beginning. After that, the vehicle navigates with integrated navigation mode to the end. In this process, it is assumed that detecting whether GNSS signal can be used or not, and switching navigation node, are accomplished by another algorithm. The detailed flight profile is shown in Table 1.

The ground surface has a slope angle of 5 degrees, after 150 meters in an easterly direction, and -7 degrees between -100 meters and 80 meters in an easterly direction, at the end of the trajectory of the vehicle, as shown in Fig. 6. When generating height distribution, the horizontal position resolution is set to 0.1 meters.

The total simulation time is set to 75 seconds, and during the beginning 10 seconds, an initial alignment process is conducted, to calculate the initial attitude, and estimate biases of the IMU measurements. GNSS signal outage occurs from 10 seconds after the vehicle starts moving, to the end. In this process, bias values and the covariance matrix that are estimated just before the GNSS signal blockage occurs are used as the initial bias values and covariance matrix in the proposed system. The IMU operates at $100 \mathrm{~Hz}$, and the vision sensor and LiDAR provide their measurements at $5 \mathrm{~Hz}$. Specifications of the sensors are presented in Table 2 below.

\subsection{Results}

In the simulation, the initial alignment is done in advance, to generate the initial bias and covariance estimate value. Fig. 7 shows the horizontal position estimate results. In the case of the INS only system, the error of the estimate gradually increases and diverges as time goes on, after the GNSS signal outage occurs. In particular, the error in an easterly direction is beyond 200 meters. On the other hand, the IMU/Vision/ Lidar integrated system tracks the reference trajectory well, and its divergent characteristic becomes much weaker than the other. This is because the optical flow vectors and LiDAR measurements are utilized, for estimating the INS errors in the integrated navigation filter.

The altitude estimate results are very similar to the

Table 2. Sensor data specification

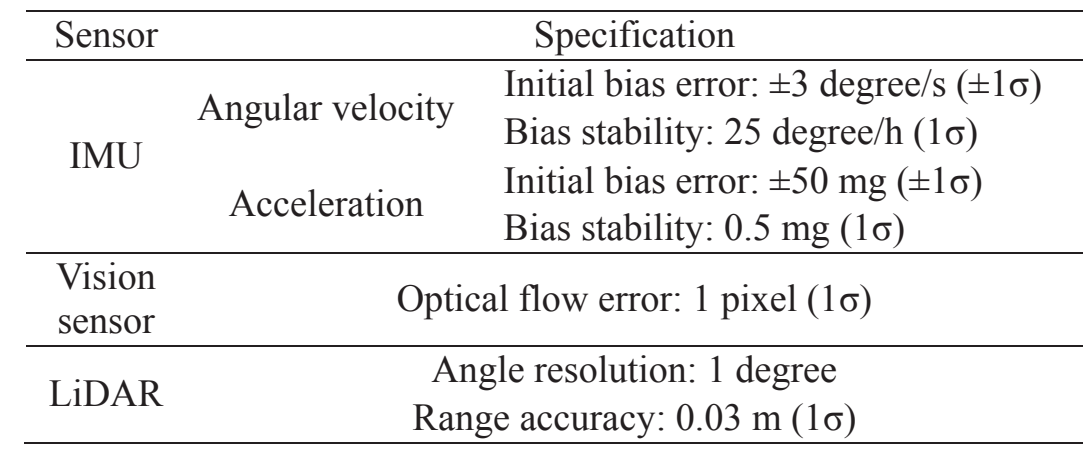

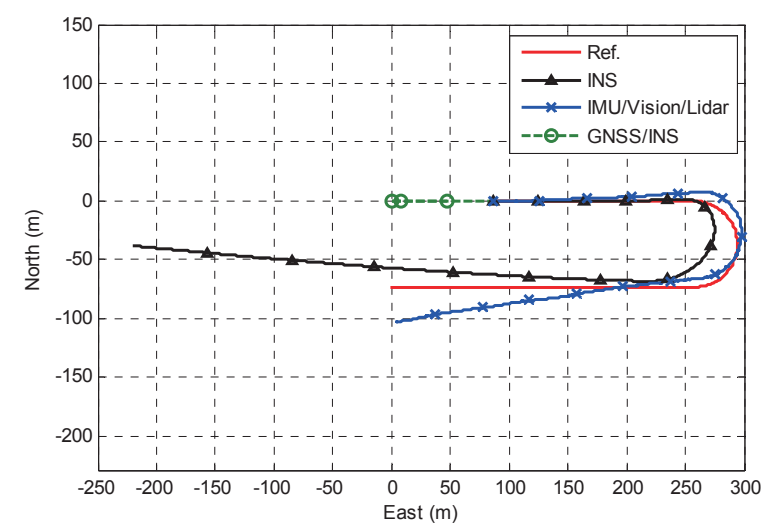

Fig. 7. Horizontal position estimate results

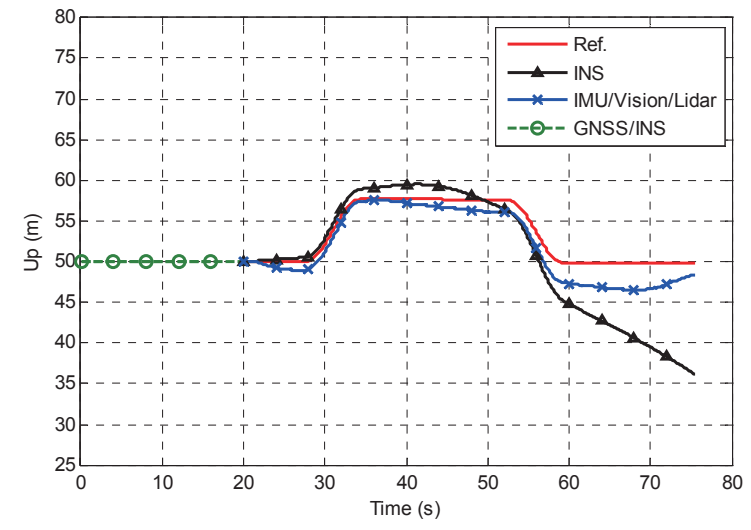

Fig. 8. Vertical position estimate results 
horizontal position estimate results. Fig. 8 shows the vertical position estimate results. In this graph, the INS error diverges, as time goes on; whereas the integrated system shows reliable estimation performance. Despite its good estimation performance, the estimation error grows slightly during short intervals of around $25 \mathrm{sec}$ and $60 \mathrm{sec}$, where the error in range measurement increases notably. This occurs when the LiDAR heads the intersection regions, in which the flat surface and sloping surface coexist at around 25 sec, and the flat surface and two sloping surfaces coexist at around $60 \mathrm{sec}$. Measurement errors are relatively amplified, as the angle between the sloping surface and the LiDAR measurements gets smaller. Furthermore, the horizontal position resolution, when generating the height distribution of the ground surface, is correspondingly degraded.

Fig. 9 shows the velocity estimate results. In common with the position estimate results, the performance of the integrated system is better than that of the INS only system.

Quantitative error analysis of the estimated position/ velocity is presented in Table 3. Root mean square errors (RMSE) of the estimated position/velocity are calculated, using the simulated true values.

Fig. 10 shows estimate results of the slope angle and height of the ground surface. The estimated slope angle is not the slope angle of the real ground surface, but that of the virtual ground surface. Thus, in the intersection areas, the estimated slope is different from the true slope. Also, the characteristic of the altitude measurement model is reflected in the estimation result of the height of ground surface. In this graph, the error of the estimated height of the ground surface becomes slightly larger, since the LiDAR measurement error and the error of the estimated forward velocity affect height variation of the ground surface.

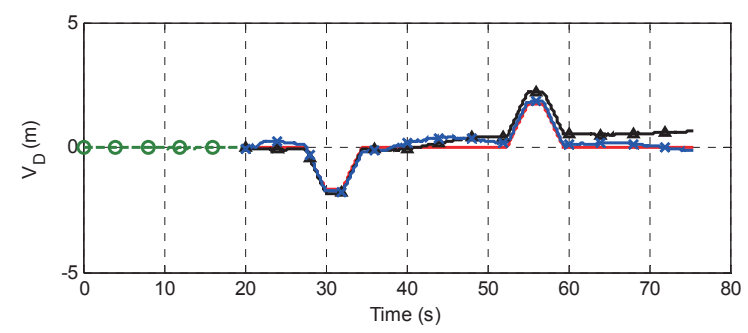

Fig. 9. Velocity estimate results
In this simulation, the integrated filter cannot estimate all state variables, due to the limitation of the flight maneuvers, which can affect the observability of the system. The proposed system can be modeled as PWCS (Piece-Wise Constant Systems), and their observability can be analyzed by SOM (Stripped Observability Matrix) rank analysis. Since observable state variables and their linear combinations are determined according to the maneuvering mode of a vehicle, the proposed system can be a fully observable system, when longitudinal and lateral acceleration are applied to the system. Verification of this theoretical analysis result can be further investigated, via more advanced simulations and experiments.

\section{Conclusions}

In this paper, we proposed a Vision/LiDAR aided integrated navigation system, in the limited environments in which GNSS signals cannot be used, the height of the ground surface is varying and there is no map information. The proposed system consists of a MEMS IMU, a vision sensor and a LiDAR. In this system, the IMU is mounted on the vehicle body, and the vision sensor and LiDAR are mounted on a two-dimensional gimbal system, in order that those sensors always maintain the horizontality condition. The proposed system can provide reliable navigation solutions, by suppressing rapid divergence of the INS error, using

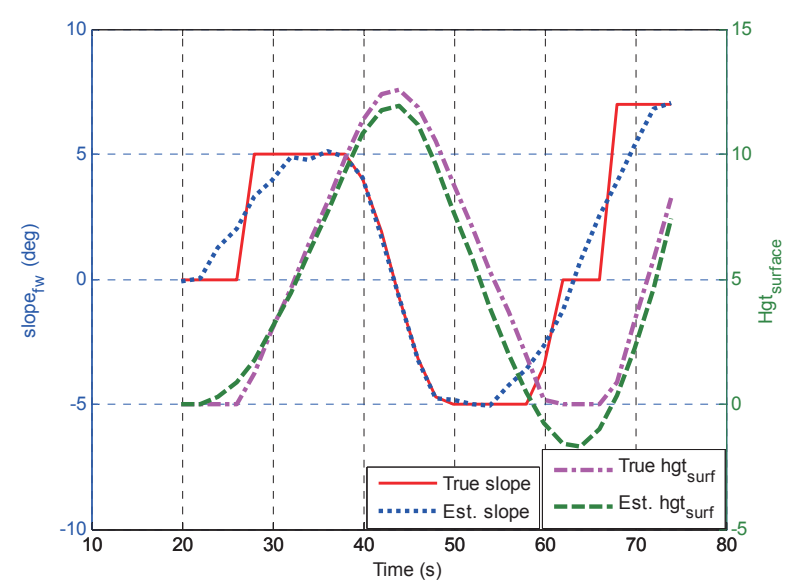

Fig. 10. Estimate result of the slope angle and height of ground surface

Table 3. Error analysis of the estimated position and velocity

\begin{tabular}{ccccccc}
\hline \multirow{2}{*}{$\begin{array}{c}\text { Navigation } \\
\text { system }\end{array}$} & \multicolumn{3}{c}{ Position error (m, RMSE) } & \multicolumn{3}{c}{ Velocity error (m/s, RMSE) } \\
\cline { 2 - 7 } & North & East & Down & North & East & Down \\
\hline INS & 13.1 & 90.8 & 5.18 & 0.86 & 4.94 & 0.38 \\
IMU/Vision/Lidar & 3.11 & 1.05 & 1.05 & 0.23 & 0.43 & 0.13 \\
\hline
\end{tabular}


vision sensor and LiDAR measurements. The horizontal velocity estimate of the INS is corrected, by using optical flow measurements from the vision sensor, and range information between the system and the ground surface, from LiDAR. At the same time, the height of the ground surface is estimated, by using forward/backward LiDAR measurements; therefore, with the estimated height, the accurate altitude can be obtained. For these purposes, an integrated navigation filter is constructed, based on the EKF framework. The feasibility of the system is proved via simulation results.

In the simulation, the performance of the proposed integrated navigation system was verified, by comparing the navigation performance of the proposed system, with that of the INS only system when GNSS signals cannot be used. As a result, our proposed integrated system provides more accurate navigation solutions than the INS. However, the problem that position error slowly increases still remains, because the system navigates relatively, without absolute navigation information, such as GNSS signals, landmarks, and so on. This problem is regarded as an inevitable limitation of a standalone navigation system.

The integrated navigation system proposed in this paper is expected to be applicable to various fields that require relative navigation information, such as a low altitude UAV that operates in the downtown area, or jamming environments, in which the GNSS availability is low.

\section{Acknowledgement}

This paper was written as part of Konkuk University's research support program for its faculty on sabbatical leave in 2012 .

\section{References}

[1] C. Becker, J. Salas, K. Tokusei, and J. C. Latombe, "Reliable Navigation Using Landmarks", Proceedings of IEEE International Conference on Robotics and Automation, Nagoya, Aichi, 1995, Vol.1, pp. 401-406.

[2] C. S. Sharp, O. Shakernia and S.S. Sastry, "A Vision System for Landing an Unmanned Aerial Vehicle", Proceedings of IEEE International Conference on Robotics and Automation, 2001, Vol.2, pp. 1720-1727.

[3] L. S. Coelho and M.F.M. Campos, "Pose Estimation of Autonomous Dirigibles Using Artificial Landmarks", Proceedings of IEEE International Conference on Robotics and Automation, Campinas, Brazil, 1999, pp. 161-170.

[4] H. Durrant-Whyte and T. Bailey, "Simultaneous
Localization and Mapping: Part I", IEEE Robotics \& Automation Magazine, 2006, Vol.13, Issue2, pp. 99-110.

[5] T. Bailey and H. Durrant-Whyte, "Simultaneous Localization and Mapping (SLAM): Part II", IEEE Robotics \& Automation Magazine, 2006, Vol.13, Issue3, pp. 108-117.

[6] J. Kim, and S. Sukkarieh, "Real-time Implementation of Airborne Inertial-SLAM”, Robotics and Autonomous Systems, 2007, Vol.55, Issue1, pp. 62-71.

[7] S. Ahrens, D. Levine, G. Andrews and J.P. How, "Vision-based guidance and control of a hovering vehicle in unknown, GPS-denied environments", Proceedings of IEEE International Conference on Robotics and Automation, Kobe, Japan, 2009, pp. 2643-2648.

[8] M. K. Kaiser, N. R. Gans and W. E. Dixon, "VisionBased Estimation for Guidance, Navigation, and Control of an Aerial Vehicle", IEEE Transactions on Aerospace and Electronic Systems, 2010, Vol.46, Issue3, pp. 1064-1077.

[9] S. Yun, B. Lee, Y. J. Lee and S. Sung "Real-Time Performance Test of an Vision-based Inertial SLAM", Proceedings of International Conference on Control, Automation and Systems, 2010, Gyeonggi-do, Korea, pp. 2423-2426.

[10] T. Cornall and G. Egan, "Optic flow methods applied to unmanned air vehicles", Academic Research Forum, Dept. Elect. And Computer Systems Engineering, Monash University, Feb, 2003.

[11] A. Giachetti, M. Campani and V. Torre, "The Use of Optical Flow for Road Navigation", IEEE Transactions Robotics and Automation, 1998, Vol. 14, Issue1, pp. 34-48.

[12] W. Ding, J. Wang, S. Han, A. Almagbile, M. A. Garratt, A. Lambert, and J. J. Wang, "Adding Optical Flow into the GPS/INS Integration for UAV navigation", International Global Navigation Satellite Society Symposium, 2009, Surfers Paradise, Qld, Australia.

[13] S. Zingg, D. Scaramuzza, S. Weiss, and R. Siegwart, "MAV Navigation through Indoor Corridors Using Optical Flow", Proceedings of IEEE International Conference on Robotics and Automation, Anchorage, AK, 2010, pp. 33613368.

[14] F. Kendoul, I. Fantoni, and K. Nonami, "Optic FlowBased Vision System for Autonomous 3D Localization and Control of Small Aerial Vehicles", Robotics and Autonomous Systems, 2009, Vol.57, Issue 6-7, pp. 591-602.

[15] S. Kohlbrecher, J. Meyer, O. von Stryk, and U. Klingauf, "A Flexible and Scalable SLAM System with Full 3D Motion Estimation", IEEE International Symposium on Safety, Security, and Rescue Robotics, 2011, Kyoto, Japan, pp. 155160.

[16] C. Premebida, O. Ludwig and U. Nunes, "LIDAR and Vision-Based Pedestrian Detection System", Journal of Field 
Robotics, 2009, Vol.26, No. 9, pp. 696-711.

[17] L. Huang and M. Barth, "Tightly-Coupled LIDAR and Computer Vision Integration for Vehicle Detection", IEEE Intelligent Vehicles Symposium, Xi'an, China, 2009, pp. 604609.

[18] P. Moghadam, W. S. Wijesoma, and J. F. Dong, "Improving Path Planning and Mapping Based on Stereo Vision and Lidar", Proceedings of International Conference on Control, Automation, Robotics and Vision, Hanoi, Vietnam,
2008, pp. 384-389.

[19] W. Ding, Optimal Integration of GPS with Inertial Sensors: Modelling and Implementation, Ph.D. thesis, University of New South Wales, Sydney, 2008.

[20] Ribeiro, M., "Kalman and extended Kalman filters: concept, derivation and properties", Technical Report, Institute for Systems and Robotics-Instituto Superior Tecnico, Lisbon, 2004.

\section{Appendix A}

Detailed expression for the system matrix from equation (10):

$\begin{aligned} F_{11} & =\left[\begin{array}{ccccccccc}0 & -\dot{\lambda}_{s} L & \dot{L} & 1 & 0 & 0 & 0 & 0 & 0 \\ \dot{\lambda} s L & 0 & \dot{\lambda} c L & 0 & 1 & 0 & 0 & 0 & 0 \\ -\dot{L} & -\dot{\lambda} c L & 0 & 0 & 0 & 1 & 0 & 0 & 0 \\ -g / R_{e} & 0 & 0 & 0 & -\left(2 \omega_{i e}+\dot{\lambda}\right) s L & \dot{L} & 0 & -f_{D} & f_{E} \\ 0 & -g / R_{e} & 0 & \left(2 \omega_{i e}+\dot{\lambda}\right) s L & 0 & \left(2 \omega_{i e}+\dot{\lambda}\right) c L & f_{D} & 0 & -f_{N} \\ 0 & 0 & 2 g / R_{e} & -\dot{L} & -\left(2 \omega_{i e}+\dot{\lambda}\right) c L & 0 & -f_{E} & f_{N} & 0 \\ 0 & 0 & 0 & 0 & 0 & 0 & 0 & -\left(\omega_{i e}+\dot{\lambda}\right) s L & \dot{L} \\ 0 & 0 & 0 & 0 & 0 & 0 & \left(\omega_{i e}+\dot{\lambda}\right) s L & 0 & -\left(\omega_{i e}+\dot{\lambda}\right) c L \\ 0 & 0 & 0 & 0 & 0 & 0 & -\dot{L} & \left(\omega_{i e}+\dot{\lambda}\right) c L & 0\end{array}\right] \\ F_{12} & =\left[\begin{array}{c}0(3 \times 3) \\ C_{b}^{n} \\ 0(3 \times 3)\end{array}\right], \quad F_{13}=\left[\begin{array}{c}0(3 \times 3) \\ 0(3 \times 3) \\ -C_{b}^{n}\end{array}\right], \quad F_{22}=F_{33}=\operatorname{diag}\left[\begin{array}{lll}0 & 0 & 0\end{array}\right]\end{aligned}$

where,

$\lambda, L$ : longitude and latitude, respectively.

g: gravity

$R_{e}$ : Earth radius

$\omega_{i e}$ : Earth rate vector

$f_{N}, f_{E}, f_{D}$ : specific force with respect to navigation frame $s L=\sin (L), c L=\cos (L)$ 